\title{
A Novel and Efficient Numerical Algorithm for Solving 2D Fredholm Integral Equations
}

\author{
H. Bin Jebreen (1D \\ Department of Mathematics, College of Science, King Saud University, P.O. Box 2455, Riyadh 11451, Saudi Arabia \\ Correspondence should be addressed to H. Bin Jebreen; hjebreen@ksu.edu.sa
}

Received 25 October 2020; Revised 26 November 2020; Accepted 2 December 2020; Published 15 December 2020

Academic Editor: Hijaz Ahmad

Copyright (c) $2020 \mathrm{H}$. Bin Jebreen. This is an open access article distributed under the Creative Commons Attribution License, which permits unrestricted use, distribution, and reproduction in any medium, provided the original work is properly cited.

A novel and efficient numerical method is developed based on interpolating scaling functions to solve 2D Fredholm integral equations (FIE). Using the operational matrix of integral for interpolating scaling functions, FIE reduces to a set of algebraic equations that one can obtain an approximate solution by solving this system. The convergence analysis is investigated, and some numerical experiments confirm the accuracy and validity of the method. To show the ability of the proposed method, we compare it with others.

\section{Introduction}

In this work, we focus on developing the wavelet Galerkin scheme for 2D Fredholm integral equations (2D-FIE), given by

$$
w(x, y)-\int_{0}^{1} \int_{0}^{1} k(x, y, s, t) g(w(s, t)) \mathrm{d} s \mathrm{~d} t=f(x, y), \quad(x, y) \in \mathscr{D},
$$

where the functions $k(x, y, s, t)$ and $f(x, y)$ are continuous functions on $\mathscr{D}:=\Omega \times \Omega$ and $\mathcal{S}$ with $\mathcal{S}:=\{(x, y, s, t): x, s \in \Omega, y, t \in \Omega\}$, respectively, as prescribed before. Function $g$ is given and can be linear or nonlinear. The unknown $w(x, y)$ is sought.

Most of the mathematical models for physical phenomena use integral equations. Consequently, the application of integral equations is a momentous subject within applied mathematics. Also, this equation plays a significant role in reformulations of other mathematical problems. Principally, the Fredholm integral equation can be obtained from converting boundary value problems [1]. Equation (1) is an effective tool to model the problems that come from electromagnetic scattering, computer graphics, and aerodynamics $[2,3]$.

Many papers studied the Fredholm integral equations numerically. Among them, we can mention an effective numerical method based on block-pulse functions [3]. Effati et al. [4] utilized a neural network scheme for solving this type of integral equation. A sparse representation based on multiwavelets has been developed and utilized an efficient algorithm for solving the system of FIE [5]. In [2], numerous numerical methods are presented and investigated for solving the numerical solution of FIE. For some related works, we refer the readers to [6-8].

There are so many papers on developing and analyzing the numerical methods for solving two-dimensional FIE. In [9], a novel algorithm is developed to solve the problem using the integral mean value theorem. Kazemi et al. [10] proposed the iterative method based on quadrature formula to solve 2D-FEI. A numerical method based on developing the two-dimensional triangular orthogonal functions is applied in [11]. Aziz et al. [12] used Haar wavelet method to solve problem (1). The principal advantage of the method is that, unlike others, it does not apply numerical integration. In [13], an Euler-type method is proposed to solve considering problems. Singh et al. [14] proposed a numerical method based on Legendre scaling functions to solve the multidimensional Fredholm integral equations. In [15], an accurate scheme is developed for solving 2D Fredholm integral equation by using the cosine-trigonometric functions. In [16], an iterative algorithm based on sparse least- 
squares is used to solve the problem. For some related works, we refer the readers to [17-20].

Multiwavelets are found an interesting basis for solving a variety of equations [21-23]. Multiwavelets have some properties of wavelets, such as orthogonality, vanishing moments, and compact support. In contrast to the wavelets and biorthogonal wavelets, they can have high smoothness and high approximate order coupled with short support [24]. Contrary to biorthogonal wavelets, multiwavelets can have the high vanishing moments without enlarging their support [25]. In general, the multiwavelets are a very powerful tool for expressing a variety of operators. At the present work, we apply the interpolating scaling functions constructed in $[6,26]$.

An outline of the remaining part of the paper is as follows. In Section 2, we devote the properties of interpolating scaling functions and related projections. In Section 3, we define the wavelet Galerkin method and investigate the convergence analysis. In Section 4, we solve some numerical examples to confirm the accuracy and efficiency of the scheme and show its ability comparing with other methods. Section 5 contains a few concluding remarks.

\section{Interpolating Scaling Functions}

Let $J \in \mathbb{Z}^{+} \cup\{0\}$. We consider the uniform finite discretizations $\Omega:=[0,1]=\cup_{b \in \mathscr{B}} X_{J, b}$, where the subintervals $X_{J, b}:=\left[x_{b}, x_{b+1}\right]$ are determined by the point $x_{b}:=\left(b /\left(2^{J}\right)\right)$, with $\mathscr{B}:=\left\{0, \ldots, 2^{J}-1\right\}$. For $k \in \mathscr{R}:=\{0,1, \ldots, r-1\}$, we introduce the subspace $V_{J}^{r}$ as a space of piecewise polynomial bases of degree less than multiplicity parameter $r$ that is spanned by

$$
V_{J}^{r}:=\operatorname{Span}\left\{\phi_{j, b}^{k}:=\mathscr{D}_{2^{j}} \mathscr{T}_{b} \phi^{k}, b \in \mathscr{B}_{j}, k \in \mathscr{R}\right\} \subset L^{2}(\Omega), \quad r \geq 0,
$$

where $\mathscr{T}$ and $\mathscr{D}$ are the translation and dilation operators, respectively, and $\left\{\phi^{k}\right\}_{k \in \mathscr{R}}$ are the primal interpolating scaling bases introduced by Alpert et al. [26]. Given nodes $\left\{\tau_{k}\right\}_{k \in \mathscr{R}}$, which are the roots of Legendre polynomial of degree $r$, the interpolating scaling bases are defined as

$$
\phi^{k}(x)= \begin{cases}\sqrt{\frac{2}{\omega_{k}}} L_{k}(2 x-1), & x \in \Omega, \\ 0, & \text { o.w }\end{cases}
$$

where $\left\{L_{k}(t)\right\}_{k \in \mathscr{R}}$ are the Lagrange interpolating polynomials at the point $\left\{\tau_{k}\right\}_{k \in \mathscr{R}}$ and $\left\{\omega_{k}\right\}_{k \in \mathscr{R}}$ are the Gauss-Legendre quadrature weights $[22,26]$. These bases form orthonormal bases on $\Omega$ with respect to the $L^{2}$-inner product. According to the definition of the space $V_{J}^{r}$, the spaces $\left\{V_{j}^{r}\right\}_{j \in \mathbb{Z}^{+} \cup\{0\}}$ have dimension $N:=2^{J} r$ and obviously are nested

$$
V_{0}^{r} \subset V_{1}^{r} \subset \cdots \subset V_{J}^{r} \subset \cdots \subset L^{2}(\Omega) .
$$

Every function $p \in L^{2}(\Omega)$ can be represented in the form

$$
p \approx \mathscr{P}_{J}^{r}(p)=\sum_{b \in \mathscr{B}_{J}} \sum_{k \in \mathscr{R}} p_{J, b}^{k} \phi_{J, b}^{k}
$$

where $\mathscr{P}_{J}^{r}$ is the orthogonal projection that maps $L^{2}(\Omega)$ onto the subspace $V_{J}^{r}$. To find the coefficients $p_{J, b}^{k}$ that are determined by $\left\langle p, \phi_{J, b}^{k}\right\rangle=\int_{X} f(x) \phi_{J, b}^{k}(x) \mathrm{d} x$, we shall compute the integrals. We apply the $r$-point Gauss-Legendre quadrature by a suitable choice of the weights $\omega_{k}$ and nodes $\tau_{k}$ for $k \in \mathscr{R}$ to avoid these integrals $[5,26]$ via

$$
p_{J, b}^{k} \approx 2^{-J / 2} \sqrt{\frac{\omega_{k}}{2}} p\left(2^{-J}\left(\frac{\tau_{k}+1}{2}+b\right)\right), \quad k \in \mathscr{R}, b \in \mathscr{B}_{J} .
$$

Convergence analysis of the projection $\mathscr{P}_{J}^{r}(p)$ is investigated for the $r$-times continuously differentiable function $p \in \mathbb{C}^{r}(\Omega)[26]$.

$$
\left\|\mathscr{P}_{J}^{r}(p)-p\right\| \leq 2^{-J r} \frac{2}{4^{r} r !} \sup _{x \in[0,1]}\left|p^{(r)}(x)\right| .
$$

For the full proof of this approximation and further details, we refer the readers to [6]. Thus, we can conclude that $\mathscr{P}_{J}^{r}(p)$ converges to $p$ with rate of convergence $O\left(2^{-J r}\right)$.

Assume that the vector function $\Phi_{J}^{r}:=\left[\Phi_{r, J, 0}\right.$, $\left.\ldots, \Phi_{r, J, 2^{J}-1}\right]^{T}$ with $\Phi_{r, J, b}:=\left[\phi_{J, b}^{0}, \ldots, \phi_{J, b}^{r-1}\right]$ includes the scaling functions and called multiscaling function. Approximation (5) may be rewritten using the vector $P$ that includes entries $P_{b r+k+1}:=p_{J, b}^{k}$ as follows:

$$
\mathscr{P}_{J}^{r}(p)=P^{T} \Phi_{J}^{r}
$$

where $P$ is an $N$-dimensional vector. The building blocks of these bases construction can be applied to approximate a higher-dimensional function. To this end, one can introduce the two-dimensional subspace $V_{J}^{r, 2}:=V_{J}^{r} \times V_{J}^{r} \subset L^{2}(\Omega \times \Omega)$ that is spanned by

$$
\left\{\phi_{J, b}^{k} \phi_{J, b^{\prime}}^{k^{\prime}}: b, b^{\prime} \in \mathscr{B}_{J}, k, k^{\prime} \in \mathscr{R}\right\} .
$$

Thus, by this assumption, to derive an approximation of the function $p \in L^{2}(\Omega \times \Omega)$ by the projection operator $\mathscr{P}_{J}^{r}$, we get

$$
p(s, t) \approx \mathscr{P}_{J}^{r}(p)(s, t)=\Phi_{J}^{r T}(s) P \Phi_{J}^{r}(t),
$$

where components of the square matrix $P$ of order $N$ are obtained by

$$
P_{r b+(k+1), r b^{\prime}+\left(k^{\prime}+1\right)} \approx 2^{-J} \sqrt{\frac{\omega_{k}}{2}} \sqrt{\frac{\omega_{k^{\prime}}}{2}} p\left(2^{-J}\left(\widehat{\tau}_{k}+b\right), 2^{-J}\left(\widehat{\tau}_{k^{\prime}}+b^{\prime}\right)\right),
$$

where $\widehat{\tau}_{k}=\left(\left(\tau_{k}+1\right) / 2\right)$. Consider the $2 r$-th partial derivatives of $p: \Omega \times \Omega \longrightarrow \mathbb{R}$ are continuous. Utilizing this assumption, the error of this approximation can be bounded as follows:

$$
\left\|\mathscr{P}_{J}^{r} p-p\right\| \leq \mathscr{M}_{\max } \frac{2^{1-r J}}{4^{r} r !}\left(2+\frac{2^{1-J r}}{4^{r} r !}\right)
$$

where $\mathscr{M}_{\max }$ is a constant $[27,28]$. 


\section{Wavelet Galerkin Scheme (WGS)}

For the completeness of this paper, we describe the wavelet Galerkin scheme for two-dimensional Fredholm integral equation (1). In the operator form, equation (1) can be written as

$$
(I-\mathscr{K}) w=f,
$$

where $I$ is the identity operator and the Fredholm operator

$$
\mathscr{K}(w)(x, y):=\int_{0}^{1} \int_{0}^{1} k(x, y, s, t) g(w(s, t)) \mathrm{d} s \mathrm{~d} t,
$$

is assumed to be compact on $L^{2}(\mathscr{D})$.

Suppose that the unique solution of (1) can be approximated as an expansion of the interpolating scaling functions via

$$
w(x, y) \approx \mathscr{P}_{J}^{r}(w)(x, y)=\Phi_{J}^{r T}(x) W \Phi_{J}^{r}(y),
$$

where $W$ is an $N \times N$ matrix whose elements must be found and the projection operator $\mathscr{P}_{J}^{r}$ maps $L^{2}(\mathscr{D})$ on the subspace $V_{J}^{r}$. Also, we can write the same expansion for function $f$. These are substituted into (13), and the coefficients $W$ are determined by using the Galerkin method. For later use, introduce the residual as

$$
r_{J}^{r}:=\left(I-\lambda \mathscr{K}_{J}^{r}\right) w_{J}^{r}-f_{J}^{r},
$$

where $w_{I}^{r}:=\mathscr{P}_{J}^{r}(w), f_{I}^{r}:=\mathscr{P}_{I}^{r}(f)$, and $K_{I}^{r}:=\mathscr{P}_{I}^{r}(\mathscr{K})$. Due to the Galerkin method, it is necessitous that $\left\langle r_{I}^{r},\left[\Phi_{I}^{r}\right]_{i}\left[\Phi_{I}^{r}\right]_{j}\right\rangle=0$, for $i, j=1: N$. To derive the approximate solution, we solve the system of algebraic equations

$$
\left\langle\left(I-\lambda \mathscr{K}_{J}^{r}\right) w_{J}^{r},\left[\Phi_{J}^{r}\right]_{i}\left[\Phi_{J}^{r}\right]_{j}\right\rangle=0 .
$$

This system can be linear or nonlinear and it depends on the function $g$. The critical step of this method is how to approximate $\mathscr{K}(w)$. For this purpose, assume that

$$
k(x, y, s, t) g(w(s, t)):=\Phi_{J}^{r}(s) \Gamma(x, y) \Phi_{J}^{r}(t), \quad(x, y, s, t) \in \mathcal{S},
$$

where $\Gamma(x, y)$ is an $N \times N$ matrix whose entries are obtained by (11). Substituting (18) into (14), one can write

$$
\begin{aligned}
\mathscr{K}(w)(x, y) & :=\int_{0}^{1} \int_{0}^{1} k(x, y, s, t) g(w(s, t)) \mathrm{d} s \mathrm{~d} t \\
& =\int_{0}^{1} \int_{0}^{1} \Phi_{J}^{r}(s) \Gamma(x, y) \Phi_{J}^{r}(t) \mathrm{d} s \mathrm{~d} t \\
& =\left(I_{\phi} \Phi_{J}^{r}(1)\right)^{T} \Gamma(x, y)\left(I_{\phi} \Phi_{J}^{r}(1)\right) \\
& =\Phi_{J}^{r}(x) G \Phi_{J}^{r}(y),
\end{aligned}
$$

where $G$ is an $N \times N$ matrix and $I_{\phi}$ is the operational matrix of integral for interpolating scaling functions introduced in [22]. Due to this approximation, system (17) may be written as

$$
W-G=F,
$$

where $f(x, y):=\Phi_{J}^{r T}(x) F \Phi_{J}^{r}$.

\subsection{Convergence Analysis}

Theorem 1. Assume that $\mathscr{K}$ is a compact operator, $I-\mathscr{K}$ is injective, and the sequence $\mathscr{K}_{J}^{r}: L^{2}(\mathcal{S}) \longrightarrow L^{2}(\mathcal{S})$ is pointwise convergent to $\mathscr{K}$ and collectively compact. Then, $\left(I-\mathscr{K}_{I}^{r}\right)^{-1}$ exists and is uniformly bounded and the solution of (13) satisfies the error estimate

$$
\left\|w-w_{J}\right\| \leq \frac{\left\|\left(I-\mathscr{K}_{J}^{r}\right)^{-1}\right\|}{1-\varepsilon_{J_{0}}\left\|\left(I-\mathscr{K}_{J}^{r}\right)^{-1}\right\|}\left(\left\|w-\mathscr{P}_{J}^{r} w\right\|+\left\|\left(\mathscr{P}_{J}^{r} \mathscr{K}-\mathscr{P}_{J}^{r} \mathscr{K}_{J}^{r}\right) w\right\|\right)
$$

Proof. Motivated by the hypothesis of the theorem, one can conclude that

$$
\left\|\left(\mathscr{F}_{J}^{r}-\mathscr{F}\right) \mathscr{F}_{J}^{r}\right\| \longrightarrow 0, \quad \text { as } J \longrightarrow \infty .
$$

Using (22), for all sufficiently large $J$, we can write

$$
\left\|(I-\mathscr{K})^{-1}\left(\mathscr{K}_{J}^{r}-\mathscr{K}\right) \mathscr{K}_{J}^{r}\right\|<1,
$$

and as a consequence of this, $I-\mathscr{K}_{J}^{r}$ is reversible.

Pick $J \geq J_{0}$ such that

$$
\varepsilon_{J_{0}} \equiv \sup _{J \geq J_{0}}\left\|\mathscr{K}_{J}^{r}-\mathscr{P}_{J}^{r} \mathscr{K}_{J}^{r}\right\|<\frac{1}{\left\|\left(I-\mathscr{K}_{J}^{r}\right)^{-1}\right\|} .
$$

Due to geometric series theorem, $\left(I+\left(I-\mathscr{K}_{J}^{r}\right)^{-1}\right.$ $\left.\left(\mathscr{K}_{J}^{r}-\mathscr{P}_{J}^{r} \mathscr{K}_{J}^{r}\right)\right)^{-1}$ exists and is uniformly bounded, i.e.,

$$
\left\|\left(I+\left(I-\mathscr{K}_{J}^{r}\right)^{-1}\left(\mathscr{K}_{J}^{r}-\mathscr{P}_{J}^{r} \mathscr{K}_{J}^{r}\right)\right)^{-1}\right\| \leq \frac{1}{1-\varepsilon_{J_{0}}\left\|\left(I-\mathscr{K}_{J}^{r}\right)^{-1}\right\|} .
$$

The investigation of convergence analysis is founded by the approximation of $I-\mathscr{K}_{J}^{r}$ by $I-\mathscr{P}_{J}^{r} \mathscr{K}_{J}^{r}$,

$$
\begin{aligned}
I-\mathscr{P}_{J}^{r} \mathscr{K}_{J}^{r} & =\left(I-\mathscr{K}_{J}^{r}\right)+\left(\mathscr{K}_{J}^{r}-\mathscr{P}_{J}^{r} \mathscr{K}_{J}^{r}\right) \\
& =\left(I-\mathscr{K}_{J}^{r}\right)\left(I+\left(I-\mathscr{K}_{J}^{r}\right)^{-1}\left(\mathscr{K}_{J}^{r}-\mathscr{P}_{J}^{r} \mathscr{K}_{J}^{r}\right)\right) .
\end{aligned}
$$

Taking norm of (26) and using (25), one can obtain

$$
\left\|\left(I-\mathscr{P}_{J}^{r} \mathscr{K}_{J}^{r}\right)^{-1}\right\| \leq \frac{\left\|\left(I-\mathscr{K}_{J}^{r}\right)^{-1}\right\|}{1-\varepsilon_{J_{0}}\left\|\left(I-\mathscr{K}_{J}^{r}\right)^{-1}\right\|} .
$$


Applying the operator $\mathscr{P}_{J}^{r}$ to both sides of (13) and then rearrange to get

$$
\left(I-\mathscr{P}_{J}^{r} \mathscr{K}_{J}^{r}\right) w=\mathscr{P}_{J}^{r} f+\left(w-\mathscr{P}_{J}^{r} w\right)+\left(\mathscr{P}_{J}^{r} \mathscr{K}-\mathscr{P}_{J}^{r} \mathscr{K}_{J}^{r}\right) w .
$$

Subtracting (28) of the original equation (13), we have $\left(I-\mathscr{P}_{J}^{r} \mathscr{K}_{J}^{r}\right)\left(w-w_{J}\right)=\left(w-\mathscr{P}_{J}^{r} w\right)+\left(\mathscr{P}_{J}^{r} \mathscr{K}-\mathscr{P}_{J}^{r} \mathscr{K}_{J}^{r}\right) w$.

Taking norm and using (27),

$$
\left\|w-w_{J}\right\| \leq \frac{\left\|\left(I-\mathscr{K}_{J}^{r}\right)^{-1}\right\|}{1-\varepsilon_{J_{0}}\left\|\left(I-\mathscr{K}_{J}^{r}\right)^{-1}\right\|}\left(\left\|w-\mathscr{P}_{J}^{r} w\right\|+\left\|\left(\mathscr{P}_{J}^{r} \mathscr{K}-\mathscr{P}_{J}^{r} \mathscr{K}_{J}^{r}\right) w\right\|\right)
$$

It is straightforward to show that $\left\|\left(\mathscr{P}_{J}^{r} \mathscr{K}-\mathscr{P}_{J}^{r} \|_{J}^{r}\right) w\right\| \longrightarrow 0$ as $J \longrightarrow \infty$. Assume that $\left\{w_{J}\right\}$ is a sequence of continuous functions so that $w_{J} \longrightarrow w$ as $J \longrightarrow \infty$. Since the orthonormal projection $\mathscr{P}_{J}^{r}$ satisfies $\left\|\mathscr{P}_{J}^{r}\right\|=1$, we can obtain

$$
\begin{aligned}
\left\|w-\mathscr{P}_{J}^{r} w\right\| & \leq\left\|w-w_{J}\right\|+\left\|w_{J}-\mathscr{P}_{J}^{r} w_{J}\right\|+\left\|\mathscr{P}_{J}^{r}\left(w-w_{J}\right)\right\| \\
& \leq 2\left\|w-w_{J}\right\|+\left\|w_{J}-\mathscr{P}_{J}^{r} w_{J}\right\| .
\end{aligned}
$$

Thus, $\forall \varepsilon>0$, there exists a number $J_{0}$ so that, for any $J_{0} \leq J$, one can write $\left\|w-w_{J}\right\| \leq(\varepsilon / 4)$. This then implies that

$$
\left\|u-\mathscr{P}_{J}^{r} w\right\| \leq \frac{\varepsilon}{2}+\left\|w_{J}-\mathscr{P}_{J}^{r} w_{J}\right\| .
$$

This implies that $\left\|w-\mathscr{P}_{J}^{r} w\right\| \leq \varepsilon$, for sufficiently large value of $J$ and because $\epsilon$ is arbitrary, consequently, $\mathscr{P}_{J}^{r} w \longrightarrow w$ as $J \longrightarrow \infty$.

\section{Numerical Examples}

To illustrate the accuracy and efficiency of the scheme, some equations of form (1) solve by the proposed method. In this section, the maximum absolute value error,

$$
e_{J}=\left|w-w_{J}\right|,
$$

and $L^{2}$-error are used to show the accuracy of the proposed method. All numerical computations are carried out simultaneously using Maple and MATLAB software.

Example 1. Let us consider the following 2D linear Fredholm integral equation:

$$
\begin{aligned}
k(x, y, s, t) & :=\frac{x}{(8+y)(1+s+t)}, \\
f(x, y) & :=(1+x+y)^{-2}-\frac{x}{48+6 y} .
\end{aligned}
$$

The exact solution is $w(x, y)=\left(1 /(1+x+y)^{2}\right)[9,11]$. The proposed method is compared with integral mean value theorem [9] and 2D-TFs method [11] in Table 1. The results in Table 1 show that the proposed method has better accuracy than other methods. Figure 1 shows the surfaces of the approximate solution and the absolute error when $r=4$ and $J=2$. In Figure 2, we show the effect of the parameters $r$ and $J$ on $L_{2}$-error. It is obvious that increasing the parameters $r$ and $J$ reduces the error. The order of convergence is presented numerically for Example 1 in Table 2. As we expected, the order of convergence must approach multiplicity $r$.

Example 2. Let us now consider the following 2D linear FIE:

$$
\begin{aligned}
k(x, y, s, t) & :=x y e^{s+t}, \\
f(x, y) & :=e^{(-x-y)} .
\end{aligned}
$$
[9].

The exact solution $w(x, y)=e^{(-x-y)}-1 / 2 x y$ is given in

Table 3 illustrates a comparison among the numerical results of the proposed method, integral mean value theorem [9], and 2D-TFs method [11]. The results show that the proposed method is better than others. Figure 3 shows the surfaces of the approximate solution and the absolute error taking $r=4$ and $J=2$. Table 4 shows the effects of the multiplicity parameter $r$ and refinement level $J$ for Example 2. The order of convergence is presented numerically for Example 2 in Table 5.

Example 3. Let us consider the nonlinear 2D-FIE given in [10]:

$$
w(x, y)=\frac{(\cos (1))^{3} x y^{2}}{36}-\frac{\cos (1) x y^{2}}{12}+\frac{x y^{2}}{18}+\sin (y)-\int_{0}^{1} \int_{0}^{1} \frac{x y^{2} s}{6} w^{3}(s, t) \mathrm{d} s \mathrm{~d} t
$$


TABLE 1: Comparison of maximum absolute value error with others for Example 1.

\begin{tabular}{|c|c|c|c|c|}
\hline \multirow{2}{*}{$r$} & \multicolumn{2}{|c|}{ WGS } & \multirow{2}{*}{$\begin{array}{c}{[11]} \\
m=n=32\end{array}$} & \multirow{2}{*}{$\begin{array}{c}{[9]} \\
m=n=32\end{array}$} \\
\hline & $r=4, J=3$ & $r=3, J=4$ & & \\
\hline$(0.2,0.2)$ & $1.12 e-06$ & $6.21 e-06$ & $9.54 e-03$ & $2.58 e-06$ \\
\hline$(0.4,0.4)$ & $3.85 e-07$ & $1.40 e-06$ & $9.03 e-03$ & $5.05 e-06$ \\
\hline$(0.6,0.6)$ & $1.44 e-07$ & $5.83 e-07$ & $1.55 e-04$ & $7.39 e-06$ \\
\hline$(0.8,0.8)$ & $1.51 e-08$ & $3.65 e-07$ & $2.62 e-04$ & $9.63 e-06$ \\
\hline
\end{tabular}

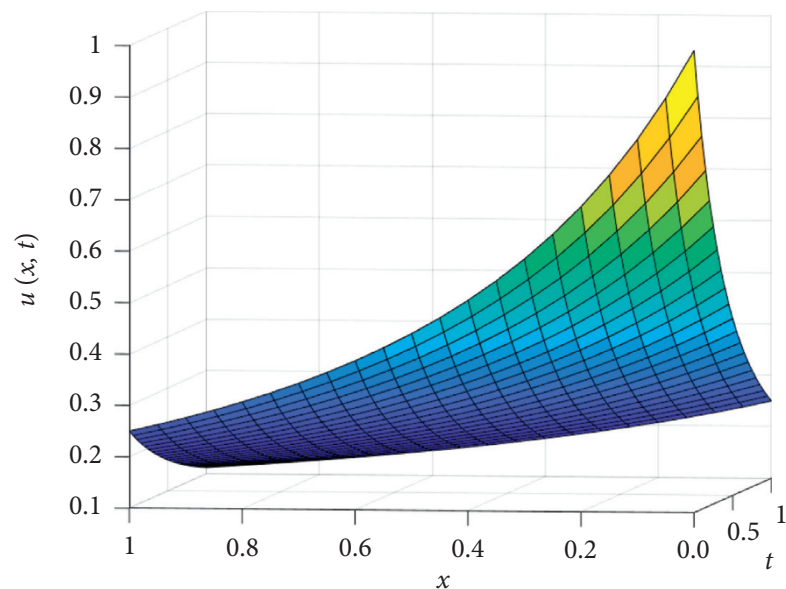

(a)

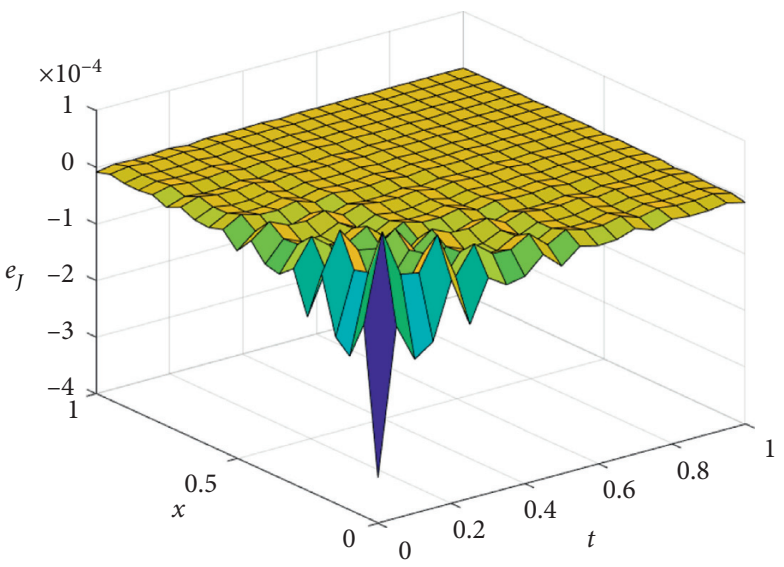

(b)

FIgURE 1: The surfaces of the approximate solution and the absolute error taking $r=4$ and $J=2$ for Example 1 .

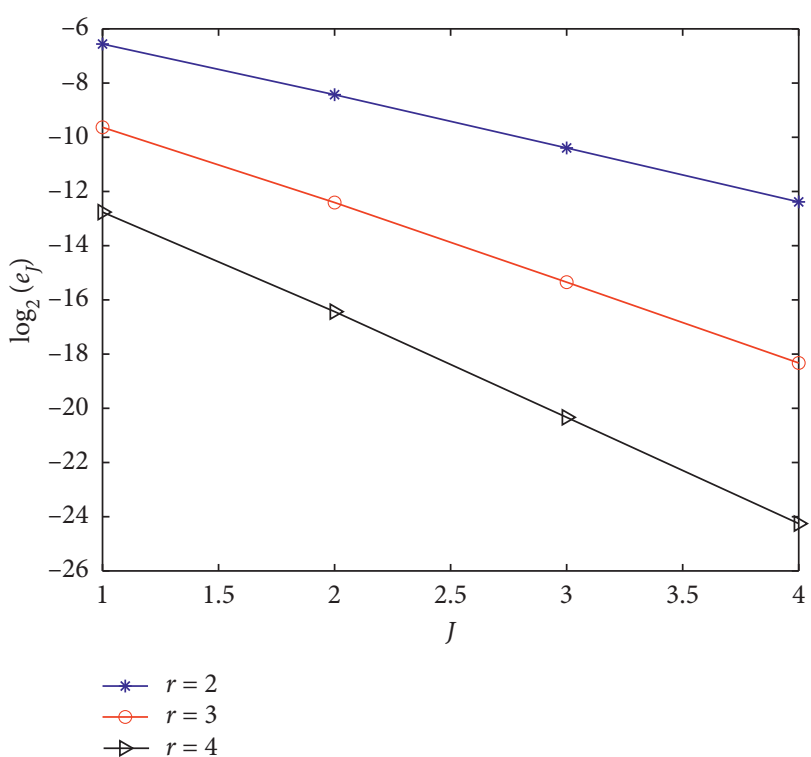

(a)

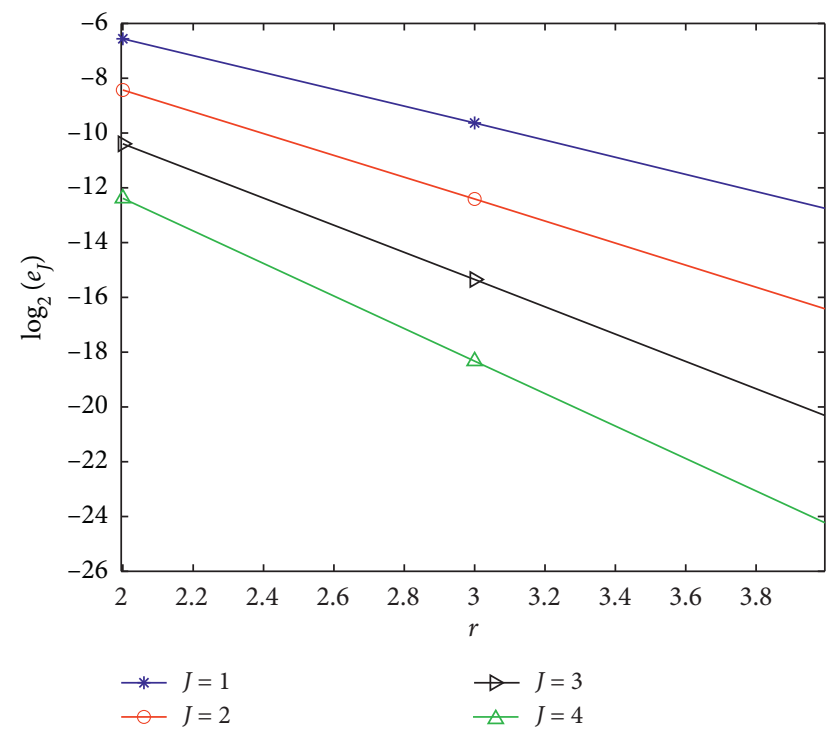

(b)

FIGURE 2: Effects of the multiplicity parameter $r$ and refinement level $J$ for Example 1.

TABLE 2: Order of convergence for Example 1.

\begin{tabular}{cccc}
\hline & $r=2$ & $r=3$ & $r=4$ \\
\hline & 1.869939460 & 2.775646062 & 3.671674134 \\
& 1.964618785 & 2.938599455 & 3.899885662 \\
& 1.990323941 & 2.980891177 & 3.920293300 \\
$\mathrm{P}$ & $\downarrow$ & $\downarrow$ & $\downarrow$ \\
\hline
\end{tabular}


TAвLE 3: Comparison of maximum absolute value errors with others for Example 2.

\begin{tabular}{lccc}
\hline$r$ & WGS & {$[11]$} & {$[9]$} \\
& $r=4, J=3$ & $m=n=32$ & $m=n=32$ \\
\hline$(0.2,0.2)$ & $9.18 e-07$ & $9.36 e-03$ & $6.49 e-06$ \\
$(0.4,0.4)$ & $2.23 e-06$ & $1.48 e-02$ & $2.60 e-05$ \\
$(0.6,0.6)$ & $4.21 e-06$ & $6.68 e-05$ & $5.84 e-05$ \\
$(0.8,0.8)$ & $6.56 e-06$ & $4.58 e-03$ & $1.04 e-05$ \\
\hline
\end{tabular}

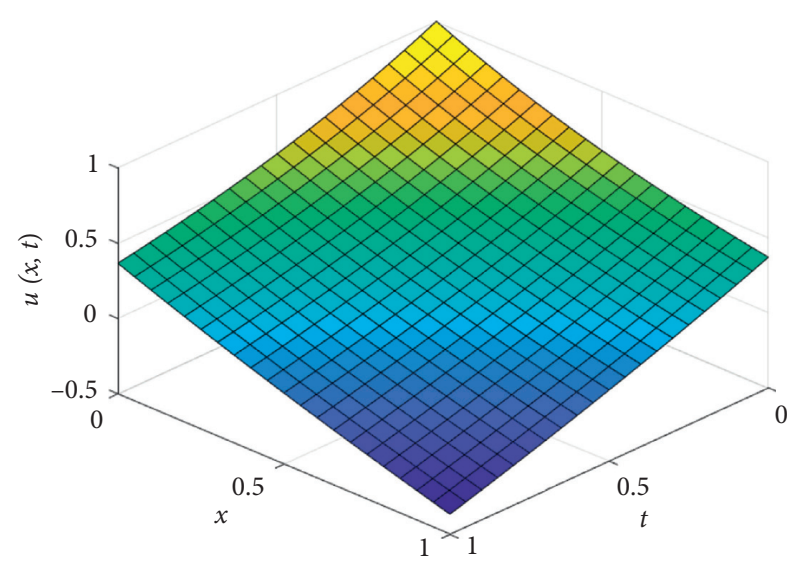

(a)

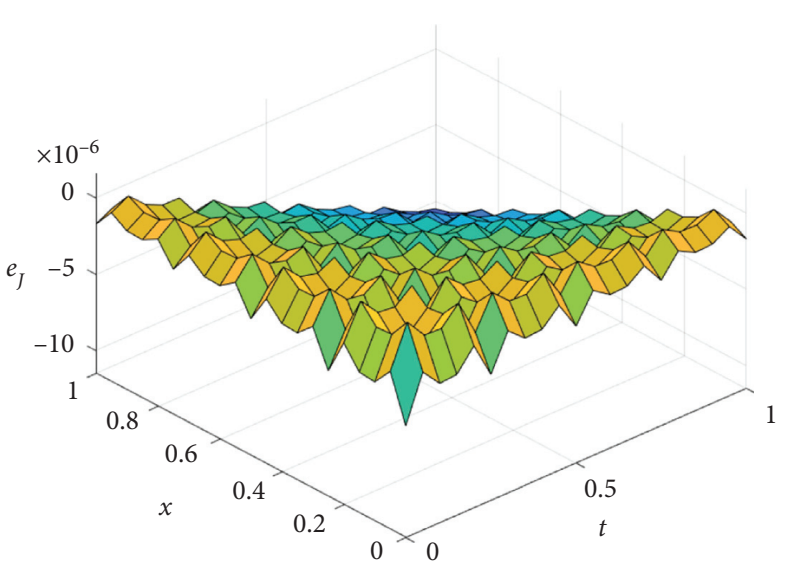

(b)

FIgURE 3: The surfaces of the approximate solution and the absolute error taking $r=4$ and $J=2$ for Example 2.

TABLE 4: Effects of the multiplicity parameter $r$ and refinement level $J$ for Example 2.

\begin{tabular}{lcccc}
\hline$r$ & $J=1$ & $J=2$ & $J=3$ & $J=4$ \\
\hline 2 & $1.46 e-02$ & $4.20 e-03$ & $1.13 e-03$ & $2.95 e-04$ \\
3 & $1.16 e-03$ & $1.54 e-04$ & $2.07 e-05$ & $2.69 e-06$ \\
4 & $5.53 e-05$ & $3.64 e-06$ & $2.45 e-07$ & $1.59 e-08$ \\
\hline
\end{tabular}

TABLE 5: Order of convergence for Example 2.

\begin{tabular}{cccc}
\hline & $r=2$ & $r=3$ & $r=4$ \\
\hline & 1.797507136 & 2.925610000 & 3.925610000 \\
& 1.894066555 & 2.885828980 & 3.893084796 \\
& 1.937535913 & 2.943952690 & 3.950893907 \\
$\mathrm{P}$ & $\downarrow$ & $\downarrow$ & $\downarrow$ \\
\hline
\end{tabular}

TABLE 6: Comparison of maximum absolute value errors with the proposed method in [10] for Example 3.

\begin{tabular}{|c|c|c|c|c|c|}
\hline & $(0.1,0.1)$ & $(0.3,0.3)$ & $(0.5,0.5)$ & $(0.7,0.7)$ & $(0.9,0.9)$ \\
\hline WGM $(r=7, J=1)$ & $1.37 e-10$ & $9.60 e-12$ & $2.98 e-10$ & $1.85 e-09$ & $3.82 e-09$ \\
\hline$[10](n=100)$ & $8.18 e-10$ & $2.21 e-08$ & $1.02 e-07$ & $2.81 e-07$ & $5.97 e-07$ \\
\hline
\end{tabular}

The exact solution $w(x, y)=\sin (y)$ is given in [10].

In Table 6, we compare the proposed method and quadrature formula proposed in [10]. It shows that the proposed method is much more flexible than the method presented in [10] and offers better accuracy using fewer computations. Figure 4 shows the plots of the approximate solution and the absolute error when $r=4$ and $J=2$. Figure 5 shows the effects of the multiplicity parameter $r$ and 


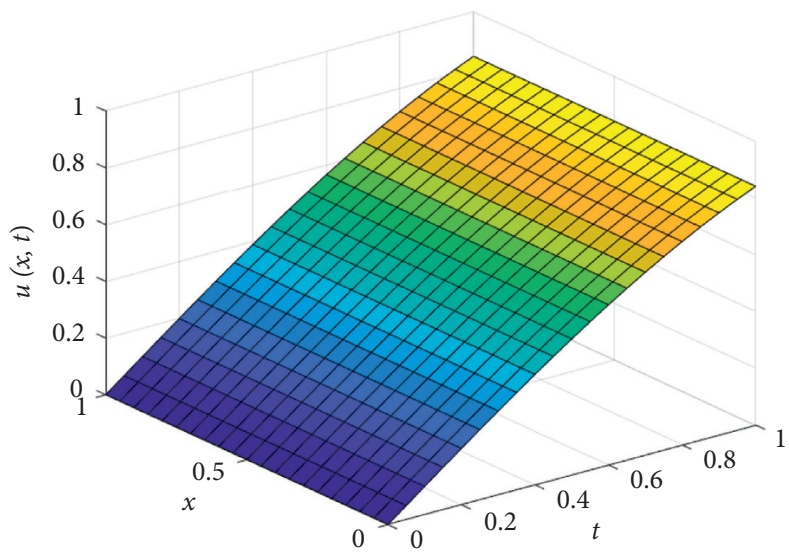

(a)

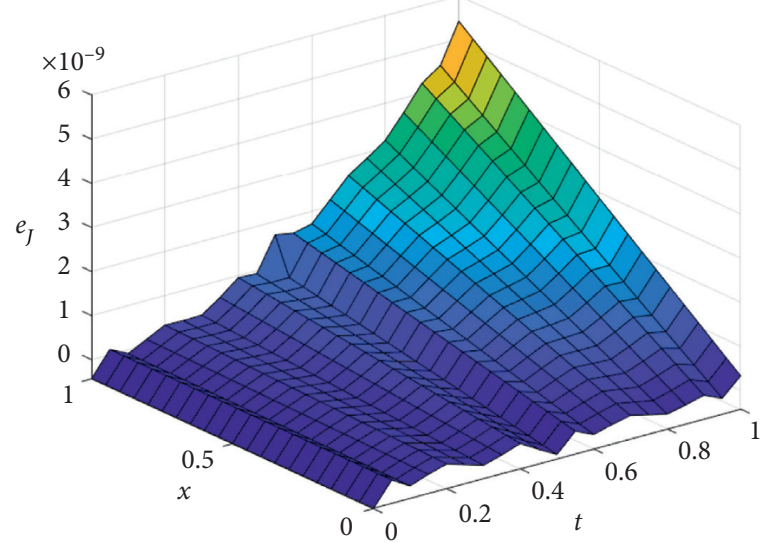

(b)

FIGURE 4: The plots of the approximate solution and the absolute error taking $r=7$ and $J=1$ for Example 3.

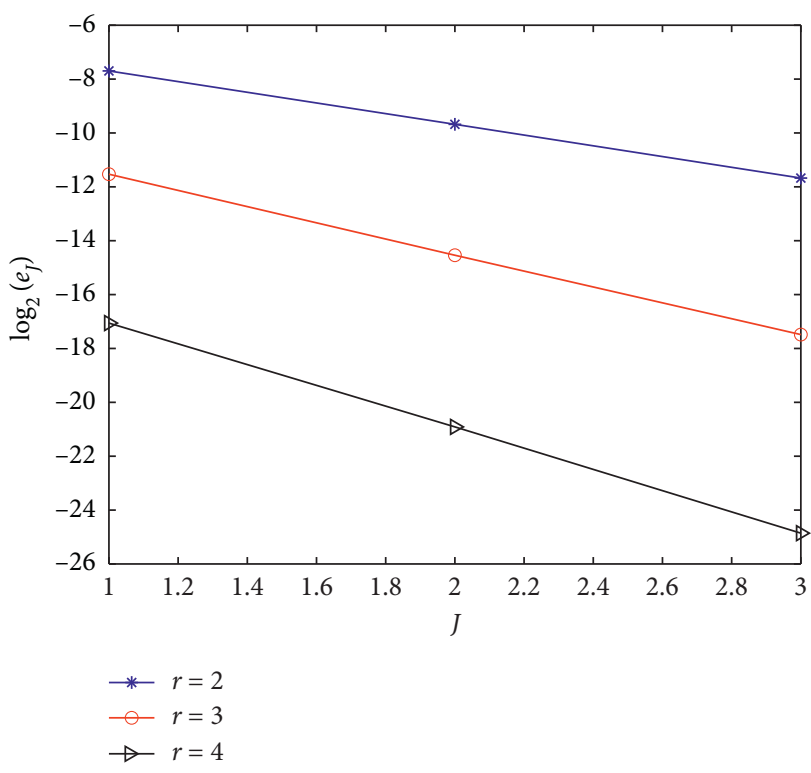

(a)

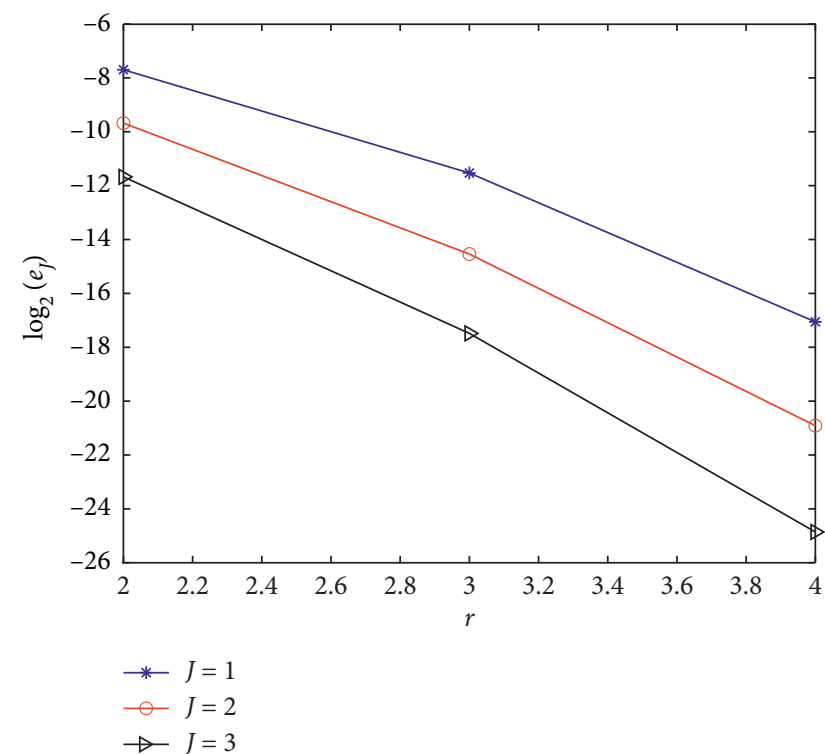

(b)

Figure 5: Effects of the multiplicity parameter $r$ and refinement level $J$ for Example 3.

TABle 7: Order of convergence for Example 3.

\begin{tabular}{cccc}
\hline & $r=2$ & $r=3$ & $r=4$ \\
\hline & 1.982151999 & 3.008562014 & 3.853755546 \\
& 1.995277590 & 2.945610000 & 3.945610000 \\
& 1.997535913 & 2.963055610 & 3.972803421 \\
& $\downarrow$ & $\downarrow$ & $\downarrow$ \\
$\mathrm{P}$ & 2 & 3 & 4 \\
\hline
\end{tabular}


TABLE 8: Comparison of maximum absolute value errors with others for Example 4.

\begin{tabular}{|c|c|c|c|c|c|}
\hline$J$ & 1 & 2 & 3 & 4 & 5 \\
\hline WGS & $7.6 e-05$ & $4.7 e-06$ & $2.9 e-07$ & $1.8 e-08$ & $1.1 e-09$ \\
\hline Haar wavelet method [12] & $2.6 e-03$ & $7.3 e-04$ & $1.9 e-04$ & $5.0 e-05$ & $1.3 e-05$ \\
\hline Nystrom method [18] & $1.4 e-02$ & $3.4 e-03$ & $8.3 e-04$ & $2.1 e-04$ & $5.2 e-05$ \\
\hline
\end{tabular}

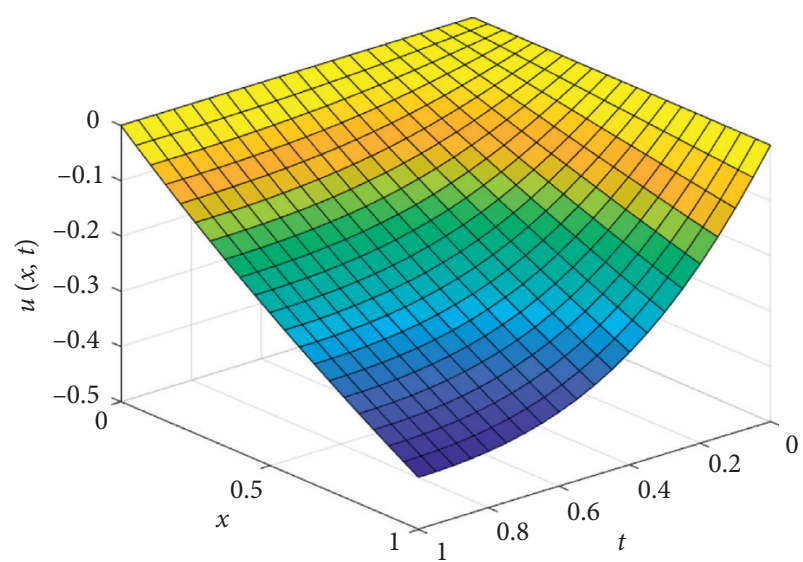

(a)

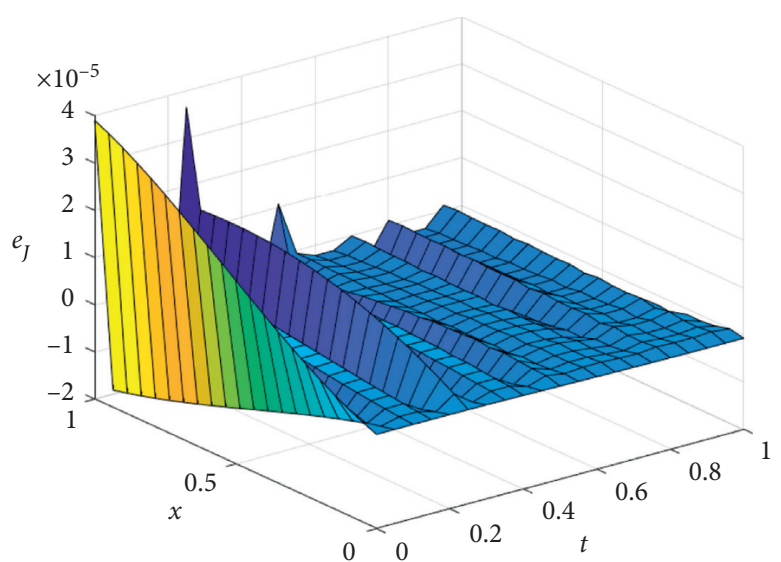

(b)

Figure 6: The plots of the approximate solution and the absolute error taking $r=4$ and $J=2$ for Example 4.

refinement level $J$ for Example 3. The order of convergence is presented numerically for Example 3 in Table 7.
Example 4. Consider the following nonlinear 2D-FIE given in $[12,18]$ :

$$
w(x, y)-\int_{0}^{1} \int_{0}^{1} \frac{x\left(1-s^{2}\right)}{(y+1)\left(t^{2}+1\right)}\left(1-e^{-w(s, t)}\right) \mathrm{d} s \mathrm{~d} t=-\log \left(1+\frac{x y}{y^{2}+1}\right)+\frac{x}{16(y+1)} .
$$

The exact solution is reported in [12] via

$$
w(x, y)=-\log \left(1+\frac{x y}{y^{2}+1}\right) .
$$

Table 8 illustrates a comparison among the numerical results of the proposed method, Nystrom method [18], and Haar wavelet method [12]. The approximate solution and the absolute error are plotted in Figure 6 when $r=4$ and $J=2$.

\section{Conclusion}

Interpolating scaling functions are applied to obtain the approximate solution of 2D Fredholm integral equations. The convergence analysis is investigated and the numerical results are compared with existing methods. It shows that the proposed method is much more flexible than others and the results show that the proposed method has better accuracy than other methods. To increase the accuracy, it is not necessary to increase the degree of polynomials (unlike bases such as Chebyshev and Legendre). By increasing the level of refinement $J$, we can increase the accuracy. Also, these bases have the interpolation property and this helps us to avoid the direct integral for finding the coefficients and this reduces the computational cost.

\section{Data Availability}

No data were used to support this study.

\section{Conflicts of Interest}

The author declares no known personal relationships or conflicts of interest that could have appeared to affect the work reported in this article.

\section{Acknowledgments}

This project was supported by Researchers Supporting Project no. RSP-2020/210 and King Saud University, Riyadh, Saudi Arabia.

\section{References}

[1] A. M. Wazwaz, Linear and Nonlinear Integral Equations: Methods and Applications, Springer Science and Business Media, Berlin, Germany, 2011.

[2] K. Atkinson, D. D. Chien, and J. Seol, "Numerical analysis of the radiosity equation using the collocation method," Electronic Transactions on Numerical Analysis, vol. 11, pp. 94-120, 2000. 
[3] S. Hatamzadeh-Varmazyar and Z. Masouri, "Numerical method for analysis of one- and two-dimensional electromagnetic scattering based on using linear Fredholm integral equation models," Mathematical and Computer Modelling, vol. 54, no. 9-10, pp. 2199-2210, 2011.

[4] S. Effati and R. Buzhabadi, "A neural network approach for solving Fredholm integral equations of the second kind," Neural Computing and Applications, vol. 21, no. 5, pp. 843$852,2010$.

[5] B. N. Saray, M. Lakestani, and M. Razzaghi, "Sparse representation of system of Fredholm integro-differential equations by using alpert multiwavelets," Computational Mathematics and Mathematical Physics, vol. 55, no. 9, pp. 1468-1483, 2015.

[6] B. Alpert, G. Beylkin, R. Coifman, and V. Rokhlin, "Waveletlike bases for the fast solution of second-kind integral equations," SIAM Journal on Scientific Computing, vol. 14, no. 1, pp. 159-184, 1993.

[7] K. E. Atkinson and F. A. Potra, "Projection and iterated projection methods for nonlinear integral equations," SIAM Journal on Numerical Analysis, vol. 24, no. 6, pp. 1352-1373, 1987.

[8] M. Lakestani, B. N. Saray, and M. Dehghan, "Numerical solution for the weakly singular Fredholm integro-differential equations using Legendre multiwavelets," Journal of Computational and Applied Mathematics, vol. 235, no. 11, pp. 3291-3303, 2011.

[9] Y. Ma, J. Huang, and H. Li, "A novel numerical method of two-dimensional fredholm integral equations of the second kind," Mathematical Problems in Engineering, vol. 2015, pp. 1-9, 2015.

[10] M. Kazemi, H. M. Golshan, R. Ezzati, and M. Sadatrasoul, "New approach to solve two-dimensional Fredholm integral equations," Journal of Computational and Applied Mathematics, vol. 354, pp. 66-79, 2019.

[11] F. Mirzaee and S. Piroozfar, "Numerical solution of the linear two-dimensional Fredholm integral equations of the second kind via two-dimensional triangular orthogonal functions," Journal of King Saud University - Science, vol. 22, no. 4, pp. 185-193, 2010.

[12] I. Aziz, S. Siraj-ul-Islam, and F. Khan, "A new method based on haar wavelet for the numerical solution of two-dimensional nonlinear integral equations," Journal of Computational and Applied Mathematics, vol. 272, pp. 70-80, 2014.

[13] S. McKee, T. Tang, and T. Diogo, "An Euler-type method for two-dimensional volterra integral equations of the first kind," IMA Journal of Numerical Analysis, vol. 20, no. 3, pp. 423440, 2000.

[14] H. Singh, D. Baleanu, H. M. Srivastava, H. Dutta, and N. Kumar Jha, "Solution of multi-dimensional fredholm equations using Legendre scaling functions," Applied $\mathrm{Nu}$ merical Mathematics, vol. 150, pp. 313-324, 2019.

[15] S. Amiri, M. Hajipour, and D. Baleanu, "On accurate solution of the Fredholm integral equations of the second kind," Applied Numerical Mathematics, vol. 150, pp. 478-490, 2020.

[16] Z. Asgari, F. Toutounian, E. Babolian, and E. Tohidi, "LSMR iterative method for solving one- and two-dimensional linear Fredholm integral equations," Computational and Applied Mathematics, vol. 38, no. 3, p. 135, 2019.

[17] I. G. Graham, "Collocation methods for two dimensional weakly singular integral equations," The Journal of the Australian Mathematical Society. Series B. Applied Mathematics, vol. 22, no. 4, pp. 456-473, 1981.
[18] H. Guoqiang and W. Jiong, "Extrapolation of Nystrom solution for two dimensional nonlinear Fredholm integral equations," Journal of Computational and Applied Mathematics, vol. 134, no. 1-2, pp. 259-268, 2001.

[19] G. Han and R. Wang, "Richardson extrapolation of iterated discrete Galerkin solution for two-dimensional Fredholm integral equations," Journal of Computational and Applied Mathematics, vol. 139, no. 1, pp. 49-63, 2002.

[20] E. Tohidi, "Taylor matrix method for solving linear two-dimensional Fredholm integral equations with piecewise intervals," Computational and Applied Mathematics, vol. 34, no. 3, p. 1117, 2014.

[21] M. Dehghan, B. N. Saray, and M. Lakestani, "Mixed finite difference and Galerkin methods for solving Burgers equations using interpolating scaling functions," Mathematical Methods in the Applied Sciences, vol. 37, no. 6, pp. 894-912, 2014.

[22] B. N. Saray, "An efficient algorithm for solving Volterra integro-differential equations based on Alpert's multi-wavelets Galerkin method," Journal of Computational and Applied Mathematics, vol. 348, pp. 453-465, 2019.

[23] S. H. Seyedi, B. Nemati Saray, and A. Ramazani, "On the multiscale simulation of squeezing nanofluid flow by a highprecision scheme," Powder Technology, vol. 340, pp. 264-273, 2018.

[24] F. Keinert, Wavelets and Multiwavelets, Chapman and Hall/ CRC, Bocaraton, FL, USA, 2003.

[25] N. Hovhannisyan and S. M. .R. Schafer, "Adaptive multiresolution discontinuous Galerkin schemes for conservation laws," Mathematics of Computation, vol. 83, pp. 113-151, 2014.

[26] B. Alpert, G. Beylkin, D. Gines, and L. Vozovoi, “Adaptive solution of partial differential equations in multiwavelet bases," Journal of Computational Physics, vol. 182, no. 1, pp. 149-190, 2002.

[27] H. Bin Jebreen, Y. Chalco Cano, and I. Dassios, "An efficient algorithm based on the multi-wavelet Galerkin method for telegraph equation," AIMS Mathematics, vol. 6, no. 2, pp. 1296-1308, 2021.

[28] B. N. Saray, "Sparse multiscale representation of Galerkin method for solving linear-mixed Volterra-Fredholm integral equations," Mathematical Methods in the Applied Sciences, vol. 43, no. 5, pp. 2601-2614, 2020 